\title{
Design and research of cutting load measuring device for coal and rock
}

\author{
Chenxu Luo', Shuangxi Jing ${ }^{2}$, Jinle Yuan ${ }^{3}$, Piao Sheng ${ }^{4}$, Lan Chen ${ }^{5}$ \\ School of Mechanical and Power Engineering, Henan Polytechnic University, Jiaozuo, 454000, P. R. China \\ ${ }^{1}$ Corresponding author \\ E-mail: ${ }^{1}$ cumtlchx@hpu.edu.cn, ${ }^{2} j i n g s h x i h p u @ 163 . c o m,{ }^{3} y u a n j l s @ f o x m a i l l . c o m,{ }^{4}$ shengpiao@hpu.edu.cn, \\ 5cljing218@163.com
}

Received 12 September 2017; accepted 18 September 2017

DOI https://doi.org/10.21595/vp.2017.19108

Check for updates

\begin{abstract}
Based on the analysis of the present situation of the research on the cutting process of the drum, the research method of the equipment capable of obtaining the cutting force in the drum cutting process is proposed by combining the finite element explicit dynamic analysis. Aiming at the complicated force of the shearer's pick cutter in cutting the coal rock. In this paper, the finite element software is used to explicit dynamics simulate the picking of coal mining in the cutting process, and the strain distribution on the cutting is determined. Based on this, the use of strain gauge bridge design ideas to indirectly obtain the force on the cutter, and finally get the relevant experimental data. Compared with the finite element simulation analysis, the feasibility of the two schemes is explained, and the foundation of the follow-up experiment is laid.
\end{abstract}

Keywords: pick-shaped cutter, rock and rock cutting, explicit dynamics, strain gauges, cutting loads.

\section{Introduction}

Pick-shaped cutter are helical drilling shearer, drum shearer, boring machine and other mining machinery commonly used coal cutting tool. Its load characteristics directly affect the cutting mechanism of cutting efficiency, energy consumption, reliability and other performance indicators. Therefore, domestic and foreign scholars have carried out a lot of research on pick picks. In China, Zhou You, Wang Zhengrong, Ji Guoqiang et al. [1-3] studied the influence of the installation parameters and the chip parameters on the load characteristics of the picking machine on the drum shearer and the boring machine by using the nonlinear dynamic simulation software. Li Xiaohuo, Wang Cunhua, Liu Chunsheng et al. [4-6] using experimental methods to study the linear cutting state of the load characteristics of the load, pointed out that the cutting force of the factors. In the past, scholars have done a lot of work on the research of pick-type picks. The relationship between the parameters such as cutting distance, geometric parameters, cutting parameters, coal and rock characteristics and cutting load characteristics is mainly studied. In view of this, this paper intends to use the finite element software to determine the strain distribution on the picking by explicit dynamical simulation of the coal mining process. Based on this, the strain gauge bridge is used to indirectly obtain the force on the picks, and the relevant data are finally obtained for analysis.

\section{Explicit dynamic analysis of coal-rock process with pick-type cutting establishment of cutting finite element model}

The shape and geometrical parameters of the pick shape used in this paper are shown in Fig. 1.

The finite element model of the rock cutting of drum cutting includes the finite element model of pick and the finite element model of coal and rock material. Because of the study of the load characteristics of the pick-type cutting rock and rock, it can ignore the influence of the tooth deformation. Therefore, the rigidity of the material with high efficiency and no deformation is selected. The elastic modulus is $270 \mathrm{GPa}$ and the density is $7800 \mathrm{~kg} / \mathrm{m}^{3}$, Poisson's ratio is 0.3 . The trimming grid adopts SOLID164 unit, and the unit integral form is single point Gaussian integral. The brittleness damage model (*MAT-BRITTLE-DAMAGE) has a density of $1500 \mathrm{~kg} / \mathrm{m}^{3}$, a modulus of elasticity of $1400 \mathrm{MPa}$, a Poisson's ratio of 0.25 , a tensile strength of $3 \mathrm{MPa}$, a 
compressive strength of $30 \mathrm{MPa}$, and a single point Gaussian SOLID164 unit of integral algorithm. Define the contact type between the pick and the coal and the rock as the surface erosion contact (ESTS). Due to the cutting of coal and rock during the process, the coal should be kept stationary, thus restraining the full degree of freedom of the nodes on the annular section of the coal rock relative to the tooth, and applying a non-reflective boundary to the outer surface of the cylindrical rock Condition to prevent the reflection of the stress wave at the boundary to affect the solution domain. The rotation speed and the feed rate along the axis are applied to the axis of the cylindrical coal and rock to simulate the drilling conditions of the drum.

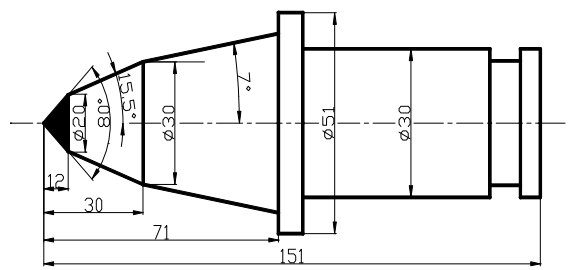

Fig. 1. Geometric parameters of pick-type picks

Cutting force is one of the measurement indicators of cutting performance. Drum picks on the drum shears are subjected to three-way cutting forces, namely cutting resistance $F_{c}$, feed resistance $F_{t}$ and radial force $F_{r}$.

After using LS-DYNA to solve the truncated coal rock cutting finite element model, the cutting force curve can be extracted. Here simulation time of $0.1 \mathrm{~s}$, extraction of 300 sampling points. Since LS-DYNA can only identify the Cartesian Cartesian coordinate system, it can only obtain the cutting force component of the pick-type trimming in three coordinate directions along the coordinate system. From the foregoing analysis, we can see that the cutting resistance of the cutting tool along the tangential movement of the tangential, feed resistance along the drum axial, it should be through the following formula to convert the cutting force:

$\left\{\begin{array}{l}F_{c}=\sqrt{F_{x}^{2}+F_{y}^{2}} \times\left|\sin \left(\omega t-\arctan \left(\frac{F_{x}}{F_{y}}\right)\right)\right|, \\ F_{t}=\left|F_{z}\right|,\end{array}\right.$

where: $F_{x}, F_{y}, F_{z}$ - cut the cutting force in the global Cartesian coordinates $X, Y, Z$ three coordinate components, $N ; \omega$ - rotation speed of rotation, rad; $t$ - time, $\mathrm{s}$.

Finally, the maximum value of the cutting load is $F_{x \max }=5000 \mathrm{~N}, F_{y \max }=8000 \mathrm{~N}$, $F_{\text {zmax }}=7000 \mathrm{~N}$ by simulation analysis.

Therefore, the measurement device design process, the need to consider the distribution of the load in three directions, cannot ignore the cutting feed resistance on the drum cutting performance and strength of the impact.

\section{Design and research of test device for cutting - type cutting}

1) Development of three - way force sensor.

The sensor is based on the rock and rock cutting table designed with the pick and tooth fixture can be effectively connected to the three-way force sensor, which has $X, Y, Z$ three signal output channels, each channel is a separate signal acquisition circuit, and any two channels of the signal will not produce mutual influence, so the authenticity of the sensor and reliability has been guaranteed.

The design of the back of the sensor can be designed to be directly connected with the tooth holder fixture form (shown in Fig. 2); in the connection with the tooth at the processing of a certain gap with the handle hole sink, so the picks can be connected to the front of the sensor via a gasket. 
This design allows the sensor structure to meet the cutting conditions, can directly obtain the cutting force data, and has a convenient replacement and installation features and functions.

The reliability of the sensor is measured and guaranteed in the process of sensor calibration. In the experiment, the finite element analysis of the force in the three directions is $0-8000 \mathrm{~N}$, and the maximum value of the force applied when calibrating the sensor is larger than that in the experiment May be subject to cutting force, so as to ensure that the sensor is not damaged during the experiment. In the end, the customized sensor force range is $0-10000 \mathrm{~N}$, the sensitivity is $1.5 \mathrm{mv} / \mathrm{V}$, the coupling force of the three-way force is less than $5 \%$, and the centrifugal output has little effect on the collected signal under the experimental conditions. The sensor uses three frame structure, can simultaneously get three-dimensional space of three forces $\left(f_{x}, f_{y}, f_{z}\right)$ information. The advantage of this sensor is in the sensitive direction of the high sensitivity, in the non-sensitive direction has a good stiffness, the smaller the degree of coupling between the dimension. The strain gauge is attached to the thin wall of the frame structure, and the bridge output of the strain gauge can reflect the force of the frame structure in the sensitive direction. The sensitive directions of the three frames are perpendicular to each other, so the three frames are able to measure three forces that are perpendicular to each other.

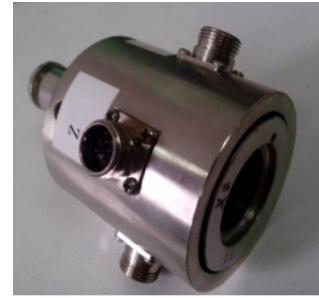

a) Trimming three-way force sensor

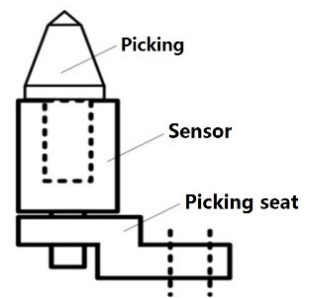

b) Sensor assembly diagram

Fig. 2. Three-way force sensor

2) Data acquisition and launch node hardware design.

Downhole practical application, the three-way force sensor channel will add a signal processing module, its main role is to sensor the signal output, amplified, into the high-precision $\mathrm{AD}$ converter into a digital signal; the digital signal through the wireless transmission Module to the wireless data receiving module; wireless transmission module using ISM band $433 \mathrm{MHz}$ frequency to send data, the transmission of high reliability, transmission distance affected by the complex terrain smaller.

In this paper, the acquisition and transmission nodes are mainly composed of three-way force sensor, power supply, signal conditioning circuit, power supply module, CC1100 wireless communication module and its related peripheral circuit. The block diagram is shown in Fig. 3(a). The information acquisition module uses the appropriate sensor to collect the useful information of the measured object, uses its peripheral circuit to carry on the level conversion and the enlargement processing to the acquisition signal, and then sends the data to the microprocessor module, the microprocessor according to the communication agreement data packing After the transmission through the RF transmitter to the data receiving node and command transmission, and then transfer the data to the host computer, the host computer for further processing and analysis of the data.

Fig. 3(b) for the acquisition node hardware diagram, the acquisition node by the sensor module, processor module, power supply module and wireless module 4 parts. Using lithium battery power supply, the normal supply voltage is $12 \mathrm{~V}$, the use of HT7333 regulator chip to provide a stable 3.3 V voltage chip, REF5040 AD conversion module to provide $2.5 \mathrm{~V}$ reference voltage. The following will be specific to the various parts of the important parameters.

As the wireless transmitter module in the transmission of data is a high-power components, will interfere with the acquisition of single-chip, it is necessary to isolate it. General isolation with 
the microcontroller RXD, TXD port isolation using optocoupler isolation, and power isolation is the use of input voltage DC4.5-9 V output DC5V isolated power supply module, the isolation method can achieve the best communication effect and Stable and reliable, the circuit schematic shown in Fig. 4.

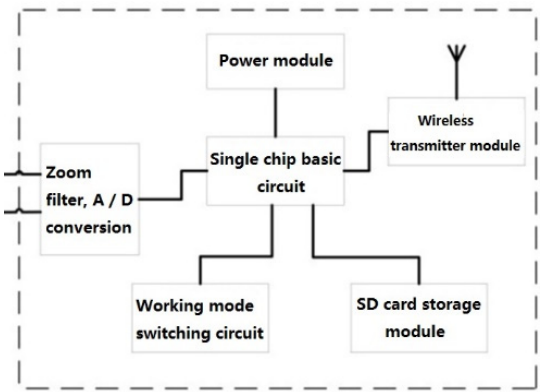

a) Structure schematic diagram

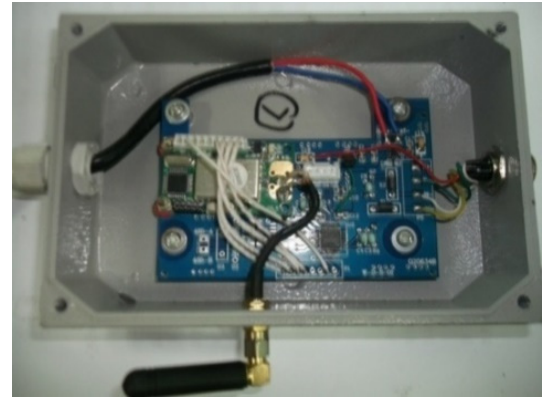

b) Physical map

Fig. 3. Acquisition and launch nodes

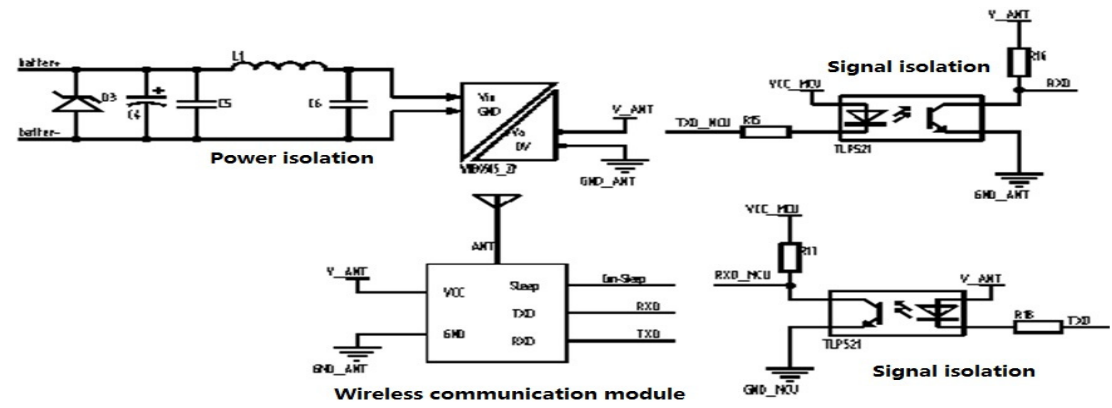

Fig. 4. SCM acquisition transmitter circuit

3) Hardware design of data receiving and instruction transmitting nodes.

The data receiving and instruction transmitting node is responsible for sending the test instruction, controls the working mode of the collecting node, receives and preliminarily processes the information sent by the collecting node. Fig. 5(a) is a physical representation of the data reception and instruction transmission nodes in this paper.

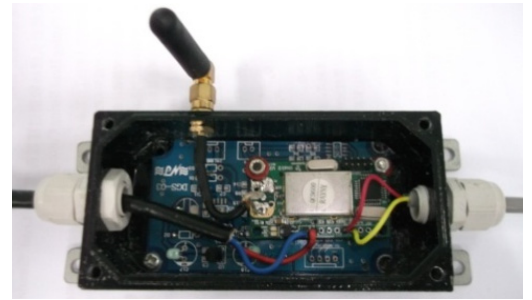

a) Physical map

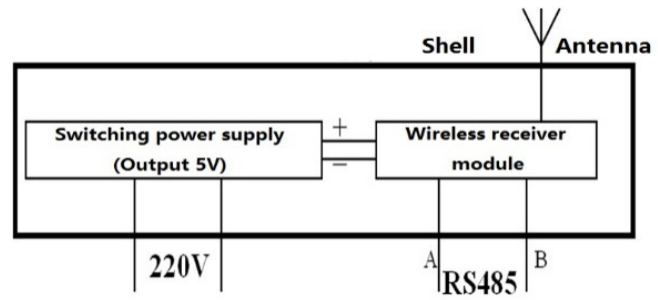

b) Circuit diagram

Fig. 5. Reception control module

As the receiving module is installed in a fixed position, it can be powered by the mains; the module is attractive in that it is based on industrial-grade narrowband wireless communication module, as long as it does not send data to the operation, it can also be used as a receiving module waiting to receive data. Since the power supply of the wireless communication module is DC5V, the switching power supply is selected to supply power to the receiving module. In addition, since the other end needs to communicate with the host computer and the communication distance may 
be far away, the RS485 port of the wireless communication module is selected as the host computer Communication interface, so that the receiving module and the host computer communication distance between up to tens of meters. Fig. 3-4(b) for the receiving module circuit design diagram.

Wireless transmission equipment and the host computer using RS-485 bus connection, can regularly upload data information to facilitate future data management and maintenance. The wireless sensor system can monitor the production status and safety of the well site, and transmit the single point condition information to the host computer, shorten the fault detection and elimination time, and greatly improve the production efficiency and economic efficiency.

4) SD card storage module.

The experimental collection device includes AVR single chip minimum system, signal adjustment amplifier module, A/D conversion module, data storage module and power module. The sampling frequency measuring device is $500 \mathrm{~Hz}$.

Connect the USB interface, the module stops receiving the stored data, automatically converted to U disk, support all file operations and formatting operations, you can remove the SD card, you can directly read the data through the card reader. SD card contains a large number of files, upload speed and formatting operation is relatively slow, before the operation, first copy the configuration file (Config.ini) to the root directory, initialize the SD card for the FAT32 file system. Before the module is powered on, start the configuration file to read the configuration information. The module can automatically identify the information stored on the SD card, support FAT file system mode. The SD card in the microcontroller program can work to obtain data, and then SD card directly through the USB interface and the host computer directly read the file directly to achieve file copy, storage and other operations. Do not turn off the power during re-storage, which may cause damage to the file.

\section{PC development}

This paper uses LabVIEW graphical software platform for testing the host computer development. The host computer has two aspects of the function: on the one hand is the wireless and wired data acquisition mode to obtain the signal storage, display operation and follow-up; the other is the next bit SD card storage of the data to read and handle operations.

1) Host computer data acquisition module.

The user interface can display the kinematic parameters such as the speed of the shearer's drum and the feed rate of the coal wall during the test. The parameters are used to cut the three-direction time-domain curve and have continuous display, peak display, etc. Multiple display modes.

The test and test system receives the digital signal sent by the signal conditioning module through the wireless receiving module, carries on the preset operation according to the data processing result, real-time displays the intercepted resistance value, remotely sets or modifies the parameters of the signal processing module Wait. According to actual needs, each three-way force sensor is equipped with three wireless signal transmission equipment and a host computer. In order to more intuitively observe the measured data, according to the requirements of simulation cutting test data collection, can be made a data acquisition system.

There are four display windows on the man-machine interaction interface. The test parameters (the moving speed of the coal wall, the rotation speed of the drum and the three-way force of the pick-type cutting, etc.) are displayed in real time during the simulation test. Use the steps: the first step to click on the collected data, according to the pop-up prompts to determine the location of the collected data; the second step when the test roller cutting simulation coal wall, click to save the data, the collected analog data will be saved to the computer specified Position and can be observed through the display interface in real time; the third step when the drum to complete the cutting test, click to stop the acquisition; Finally, click to exit, the acquisition system program to stop running, complete the test data measurement process.

2) SD card data reading module. 
When the next bit machine directly stores the cutting force signal, in order to ensure the speed and reliability of the file storage, there is no option to facilitate the analysis and processing of the file format, but in the lower computer data storage widely used in high-speed, high stability of the binary file format. So that the host computer processing data, the need for the data file format to identify and read operations.

Comparison of the two acquisition scenarios in the cutting force signal, you can evaluate the two programs, the use of wireless data transmission will be used due to the different forms of wireless modules, including data transmission and reception of the signal loss will be on the sampling frequency There are certain restrictions that can meet the immediate monitoring. For SD card storage data program, due to the sampling frequency limited conditions, can meet a large number of high-speed data collection, but the data must be read after the shutdown can be read out, real-time poor, suitable for real-time monitoring is not required condition.

\section{Conclusions}

1) Based on the finite element analysis, it is necessary to design the trimming force sensor and the drum thrust sensor according to the single cutter and roller cutting test, and select the torque sensor and the displacement sensor.

2) Taking into account the pick-type cutter rotation cutting coal rock, to determine the embedded wireless monitoring system design. This paper introduces the selection of wireless communication mode, and explains the hardware design process of data reception, instruction transmitting node and signal acquisition node which make up the wireless sensor network. The design to overcome the traditional device is not intelligent, the application site is not easy to adjust, not easy to communicate with the host computer and other shortcomings. The design can be used to guide the shearer coal production work.

3) Using the LabVIEW graphical software platform to develop the host computer data acquisition module. This paper introduces another data acquisition scheme using SD card for data storage. The simultaneous implementation of the two schemes ensures immediate monitoring of experimental data at a certain sampling frequency and data acquisition at a large number of high sampling frequencies. The host computer system can be used not only in the experimental study of this paper, but also by modifying the parameter setting to meet the experimental data acquisition of the force and vibration of the rotating machinery.

\section{References}

[1] Zhongxiao M. A. Technology of coal cutting force test device and its new progress. Mining Science and Technology, Vol. 1, 1997, p. 41-44.

[2] Zhao Yaojun Study on characteristics of three-way cutting force sensor of coal. Acta Coal Science, Vol. 25, Issue 3, 2000, p. 316-320.

[3] Liu Jia Yong, Du Chang Long, Cui Xin Xia Experimental study on the arrangement of shears in shearer drums. Journal of Central South University (Natural Science Edition), Vol. 40, Issue 5, 2009.

[4] Zhou You, Li Guoshun, Tang Jinyuan Simulation of LS_DYNA for cutting coal and rock. Journal of Engineering Design, Vol. 18, Issue 2, 2011, p. 103-108.

[5] Wang Zhengrong, Xiong Xiaoyan, Zhang Hong Based on LS-DYNA shearer pick-type cutting cutting finite element analysis. Vibration, Testing and Diagnosis, Vol. 30, Issue 2, 2010, p. 163-163.

[6] Ji Guoguo Calculation and Simulation of Cutting Process of Pick - Pick of Picking Machine. University of Technology, Taiyuan, 2008.

[7] Li Xiaofang, Yin Bofeng, Li Haibin Experimental study on cutting of pick-type picks. Journal of Liaoning Technical University, Vol. 18, Issue 6, 1999, p. 649-652.

[8] Wang Chunhua, Ding Renzheng, Li Guixuan, et al. Experimental simulation of deformation and failure process of cutting coal cutting. Journal of Coal Science, 2006, Vol. 31, 1, p. 121-124.

[9] Liu Chunsheng Fractal characteristics of cut - off resistance curve of shearer's cuttings. Journal of Coal Science, Vol. 29, Issue 1, 2004, p. 115-118.

[10] Bo Yu Numerical Simulation of Continuous Miner. West Virginia University, USA, 2005. 